Methods Human adipose-derived stem cells (hASCs) were encapsulated via a micromolding method. We first manufactured polydimethylsiloxane chips containing 1600 micromolds with a circular shape, $150 \mu \mathrm{m}$ in diameter and $100 \mu \mathrm{m}$ in depth. For cell encapsulation, a solution of $2 \%$ alginate $(w / v)$ containing 3 million of hASCs per $\mathrm{mL}$ polymer was deposited onto the chips, loaded in the moulds either by sedimentation or centrifugation and crosslinked using an agarose gel charged with $\mathrm{CaCl} 2$. The number of encapsulated cells was evaluated by a CyQUANT assay immediately and 24 hour after encapsulation. The impact of encapsulation on metabolic activity was determined by a presto blue assay 24 hours after encapsulation and 24 hour after their injection through a $26 \mathrm{G}$ needle.

Results We successfully obtained cylindrical alginate microparticles presenting a diameter of $103 \pm 0.7 \mu \mathrm{m}$. Using cell quantification, we determined that the centrifugation method allowed the encapsulation of $30333( \pm 5552)$ cells within one chip versus $6056( \pm 2862)$ by sedimentation. Cell number and metabolic activity remained stable for 24 hours after encapsulation. We also demonstrated that injection through a $26 \mathrm{G}$ needle had no impact on the viability of encapsulated cells.

Conclusions Our results show that micromolding allows hASCs encapsulation into alginate particles injectable through a $26 \mathrm{G}$ needle without impacting cell viability. Future work will focus on evaluating in vitro long-term encapsulated cell survival and functionality. In case of success, we will then consider intraarticular injection in an animal model of osteoarthritis.

Disclosure of interest None declared

\section{P112 TARGETING T-CELL TRAFFICKING IN A MURINE MODEL OF SJÖGREN'S SYNDROME}

1J Campos*, 'S Nayar, ${ }^{2} \mathrm{M}$ Chimen, ${ }^{1} \mathrm{~V}$ lannizzotto, ${ }^{1} \mathrm{HM}$ McGettrick, 'BA Fisher, ${ }^{1} \mathrm{SJ}$ Bowman, ${ }^{1} \mathrm{CD}$ Buckley, ${ }^{2} \mathrm{GE}$ Rainger, ${ }^{1} \mathrm{~F}$ Barone. ${ }^{1}$ Institute of Inflammation and Ageing; ${ }^{2}$ Institute of Cardiovascular Sciences, University of Birmingham, Birmingham, UK

\subsection{6/annrheumdis-2018-EWRR2018.127}

Introduction Salivary glands of primary Sjögren's syndrome (pSS) are characterised by complex leukocyte infiltration organised into tertiary lymphoid structures (TLS). The mechanisms regulating leukocyte trafficking into inflamed salivary glands are poorly described, but dysregulated T-cell recruitment during inflammation is believed to contribute to disease onset and chronicity. We recently described a homeostatic pathway in which a B cell-derived peptide (PEPITEM), secreted in response to adiponectin, regulates $\mathrm{T}$-cell trafficking during inflammation via sphingosyne 1 phospate activity on endothelial cells. ${ }^{1}$ Loss of this pathway by downregulation of adiponectin receptor on circulating $\mathrm{B}$ cells has been demonstrated in type 1 diabetes and rheumatoid arthritis, suggesting a potential role for PEPITEM in the pathogenesis of autoimmune diseases and indicating a role for adiponectin receptor as biomarker in autoimmune diseases. ${ }^{1}$

Objectives We aimed to investigate the efficacy of PEPITEM as an inhibitor of T-cell trafficking in an inducible animal model of salivary gland inflammation that mimics the histological features of pSS and to investigate the potential translatability of this pathway in patients with pSS.
Methods Submandibular salivary glands of C57BL/6 mice were intra-ductally cannulated with luciferase-encoding replicationdeficient adenovirus to induce TLS formation as previously described. $^{2}$ Mice were administered daily either with PBS or PEPITEM by intraperitoneal injection from day 0 , and their salivary glands dissected at day 5 post cannulation. T-cell infiltration into salivary glands was assessed using a combination of flow cytometry, immunofluorescence and qRT-PCR.

Results B cells in sera from cannulated animals express lower levels of both adiponectin receptors 1 and 2 in comparison with non-inflamed control mice. In cannulated animals treated with PEPITEM, histological analysis of salivary glands revealed fewer, as well as less aggregated, infiltrating $\mathrm{T}$ cells. Both CD4 + and CD8 + numbers were significantly lower in the salivary glands of PEPITEM-treated animals. Furthermore, administration of PEPITEM also decreased mRNA transcripts for lymphotoxin beta, IL-7, lymphoid chemokines (CCL19 and CXCL13) and T cell chemokine receptor CCR7, cytokines and chemokines known to regulate ectopic lymphoneogenesis in pSS. Human samples of pSS are currently being assessed to validate the relevance of this pathway in pSS.

Conclusions These results demonstrate that administration of exogenous PEPITEM can reduce T-cell influx into salivary glands. This may represent a rescue of the homeostatic regulation of leukocyte trafficking, which is disrupted in inflammation. Our work suggests that PEPITEM should be considered to address the therapeutic needs in chronic inflammatory conditions and that the detection of decreased levels of adiponectin receptor could be used as biomarkers in pSS.

\section{REFERENCES}

1. Chimen McGettrick, et al. Nat Med 2015.

2. Bombardieri Barone, et al. J/ 2012.

\section{Disclosure of interest None declared}

\section{P113 ARGINASE I AND THE METABOLIC CONTROL OF OSTEOCLASTOGENESIS}

${ }^{1}$ JS Brunner*, ${ }^{1} \mathrm{M}$ Hofmann, ${ }^{2} \mathrm{~V}$ Saferding, ${ }^{1} \mathrm{~A}$ Vogel, ${ }^{3} \mathrm{~A}$ Lercher, ${ }^{4} \mathrm{P}$ Cheng, ${ }^{1} \mathrm{G}$ Schabbauer, ${ }^{2} S$ Blüml. ${ }^{1}$ Institute for Vascular Biology; ${ }^{2}$ Dpt. of Rheumatology, Medical University Vienna; ${ }^{3}$ CeMM, Vienna, Austria; ${ }^{4}$ Bio Cancer Treatment International Ltd, Hong Kong, China

\subsection{6/annrheumdis-2018-EWRR2018.128}

Introduction Osteoclasts are giant, multi-nucleated cells that derive from the monocyte-macrophage linage and are regulators of bone turnover. Availability and catabolism of L-Arginine have been implicated with immune cell biology, skewing inflammatory responses within myeloid cells in a pro- or antiinflammatory manner.

Objectives While the role of L-Arginine within certain myeloid lineages such as macrophages is well appreciated, its role within osteoclasts is relatively unknown. We therefore aim to investigate L-Arginine metabolism in the context of osteoclastogenesis.

Methods We analysed osteoclastogenesis of C57BL/6J wildtype bone marrow cells in vitro in the presence and absence of recombinant Arginase 1 (recArg1). This approach was complemented via $\mathrm{qPCR}$ analysis of relevant osteoclast marker genes and an extracellular flux assay. We further investigated the 
effect of recArg1 regarding an in vivo model called serum transfer arthritis, where we treated C57BL/6J wildtype mice with the recombinant enzyme. Disease severity was then assessed using clinical scores and paw histology.

Results We observed that ARG1 mRNA expression was downregulated from the progression of a precursor to a mature osteoclast. We incubated day 3 osteoclast precursors with recArg1 and observed that addition of $1000 \mathrm{ng} / \mathrm{ml} \mathrm{recArg} 1$ abolished osteoclastogenesis. L-Arginine deprivation led to a decrease in the oxygen consumption rate of osteoclast precursor cells, assessed 48 hour after RANKL addition. Using serum transfer arthritis, an established murine in vivo model, recArg1 treated mice showed reduced disease severity combined with a significant decrease in the presence of osteoclasts. Treatment efficiency was evaluated using an L-Arginine ELISA, where the amino acid was found to be absent in the serum of treated mice.

Conclusions We propose that the amino acid L-Arginine is critical for the development of osteoclasts from myeloid precursors and hypothesise that its abundance, influenced by recArg1 addition, influences development and severity of osteoclast driven diseases.

Disclosure of interest J. Brunner Grant/research support from: Bio Cancer Treatment International Ltd, M. Hofmann Grant/ research support from: Bio Cancer Treatment International Ltd, V. Saferding Grant/research support from: Bio Cancer Treatment International Ltd, A. Vogel Grant/research support from: Bio Cancer Treatment International Ltd, A. Lercher: None declared, P. Cheng Shareholder of: Bio Cancer Treatment International Ltd, G. Schabbauer Grant/research support from: Bio Cancer Treatment International Ltd, S. Blüml Grant/research support from: Bio Cancer Treatment International Ltd

\section{P114 IONISING RADIATION INHIBITS INFLAMMATION IN PATIENTS WITH MUSCULOSKELETAL DISEASES: RADON TREATMENT VS LOW-DOSE RADIATION THERAPY}

${ }^{1} \mathrm{~K}$ Shreder*, 'A Cucu, ${ }^{1} \mathrm{D}$ Kraft, 'S Lehrian, ${ }^{1} \mathrm{~J}$ Kondol, ${ }^{2} \mathrm{G}$ Klein, ${ }^{3} \mathrm{~B}$ Frey, ${ }^{3} \mathrm{U}$ Gaipl, ${ }^{1} \mathrm{C}$ Fournier. 'GSI Helmholtzzentrum für Schwerionenforschung GmbH, Darmstadt; ${ }^{2}$ Association for Spa Research and Medical Practice for Cardiology, Bad Steben; ${ }^{3}$ Department of Radiation Oncology, Universitätsklinikum Erlangen, Erlangen, Germany

\subsection{6/annrheumdis-2018-EWRR2018.129}

Introduction Rheumatoid arthritis (RA) and osteoarthritis (OA) are the most common musculoskeletal diseases (MSD) that affect the joints. Reduced mobility and quality of life are the consequences of the cartilage and bone tissue destruction and the chronic inflammation process, caused by release of bone destruction markers and inflammatory factors including adipokines in the joint. Besides medications, an additional pain relief is achieved by the treatment of patients with low-dose ionising radiation, either as local low-dose radiation therapy (LDRT) or whole-body exposure to radon in radon baths or galleries.

Objectives In the previous work we showed the decrease of serum levels of visfatin and serum carboxy-terminal collagen crosslinks of type-I collagen (CTX-I) in patients treated in radon baths. ${ }^{1}$ In the present study, we analysed serum samples of patients with MSD, who had been treated locally with photon radiation (LDRT). In addition, we analysed differentiation and activity of osteoclasts that were differentiated in vitro from patient-derived monocytes.

Methods Serum samples were collected from patients before and after treatment. Levels of visfatin and CTX-I were measured by ELISA. Monocytes were isolated from blood samples of patients and cultivated with M-CSF and RANKL on bone slices for 2 weeks. Osteoclasts were defined as TRAP and Factin positive cells. TRAP activity was measured in the cell supernatants using TRAP Staining Kit.

Results In the serum of patients treated with LDRT, a trend to reduced concentration of CTX-I was observed directly after the therapy. Further, osteoclasts, differentiated in vitro from LDRT patient-derived monocytes, showed reduced TRAP activity.

Conclusions The observations made in this study so far substantiate that the radiation-induced decrease of CTX-I levels could be one main factor that is related to the attenuation of inflammation and to the decrease of disease activity in the patients with MSD. This hypothesis is endorsed by the observed reduced differentiation and activity of in vitro cultivated patient-derived osteoclasts.

\section{REFERENCE}

1. Cucu Shreder, et al. Front. Immunol 2017;8:882.

Acknowledgements This work was supported by the German Federal Ministry of Education and Research (grant no. 02NUK017A and 02NUK017G, GREWIS), by Landesamt für Gesundheit und Lebensmittelsicherheit Bayern, and by Bayrisches Staatsbad Bad Steben GmbH.

Disclosure of interest None declared

\section{P115 LOW DOSE RADIATION HAS A POSITIVE IMPACT ON BONE METABOLISM IN AN EXPERIMENTAL MODEL OF INFLAMMATORY ARTHRITIS}

'L Deloch*, 'M Rückert, ${ }^{2} \mathrm{AJ}$ Hueber, ${ }^{2} \mathrm{M}$ Herrmann, ${ }^{1} \mathrm{R}$ Fietkau, ${ }^{1} \mathrm{~B}$ Frey, ${ }^{1}$ US Gaipl. ${ }^{1}$ Department of Radiation Oncology; ${ }^{2}$ Internal Medicine 3, Rheumatology and Immunology, Universitätsklinikum Erlangen, Friedrich-Alexander-Universität Erlangen-Nürnberg, Erlangen, Germany

\subsection{6/annrheumdis-2018-EWRR2018.130}

Introduction Rheumatoid arthritis (RA) is, next to inflammation and infiltration of activated immune cells into the synovial joint, characterised by a progressive destruction of cartilage and bone. Although today's treatment options are very effective for many patients, not all of them respond properly or have to reduce medications due to adverse effects. In these patients it is crucial to slow down bone loss and inflammation in a timely manner to prevent further damage. Here, low-dose radiotherapy (LD-RT) could be an option, as it has been shown to ameliorate inflammation and to reduce pain. Using the human $T N F \alpha$ transgenic (hTNF $\alpha$ tg) mouse model as an experimental model of inflammatory arthritis, we revealed that locally applied LD-RT attenuates inflammation in the joints.

Objectives As little is known about the impact of LD-RT on bone metabolism, we thus focused on the effects of LD-RT on bone homeostasis.

Methods Bone marrow-derived osteoclasts (OC) of hTNF $\alpha$ tg mice were differentiated using M-CSF and RANK-L and 